https://helda.helsinki.fi

\title{
Understanding the role of lipids in signaling through atomistic and multiscale simulations of cell membranes
}

\section{Manna, Moutusi}

2019

Manna , M , Nieminen , T \& Vattulainen , I 2019 , ' Understanding the role of lipids in signaling through atomistic and multiscale simulations of cell membranes ' , Annual Review of Biophysics , vol. 48 , pp. 421-439 . https://doi.org/10.1146/annurev-biophys-052118-115553

http://hdl.handle.net/10138/313972

https://doi.org/10.1146/annurev-biophys-052118-115553

submittedVersion

Downloaded from Helda, University of Helsinki institutional repository.

This is an electronic reprint of the original article.

This reprint may differ from the original in pagination and typographic detail.

Please cite the original version. 


\title{
Understanding the Role of Lipids in Signaling through Atomistic and Multiscale Simulations of Cell Membranes
}

\author{
Moutusi Manna, ${ }^{1}$ TuomoNieminen, ${ }^{2}$ and Ilpo Vattulainen ${ }^{2,3}$
}

\author{
${ }^{1}$ Department of Chemistry, Indian Institute of Science Education and Research Bhopal, \\ Bhopal By-pass Road, Bhauri, Bhopal 462 066, MP, India \\ ${ }^{2}$ Laboratory of Physics, Tampere University of Technology, POB 692, FI-33101, Tampere, \\ Finland \\ ${ }^{3}$ Department of Physics, POB 64, FI-00014 University of Helsinki, Finland \\ Web: https://www.helsinki.fi/en/researchgroups/biophysics \\ E-mail:Ilpo.Vattulainen@helsinki.fi
}

Keywords: Signaling, lipids, molecular dynamics, computer simulations, multiscale simulations

\begin{abstract}
Cell signaling controls essentially all cellular processes. While it is often assumed that proteins are the key architects coordinating cell signaling, recent studies have shown more and more clearly that lipids are also involved in signaling processes in a number of ways. Lipids do, for instance, act as messengers, modulate membrane receptor conformation and dynamics, and control membrane receptor partitioning. Further, through structural modifications such as oxidation, the functions of lipids as part of signaling processes can be modified. In this context, in this article we discuss the understanding recently revealed by atomistic and coarse-grained computer simulations of nanoscale processes and underlying physicochemical principles related to lipids' functions in cellular signaling.
\end{abstract}

Preprint version of the paper, prior to submission and prior to review. The final (reviewed version of the paper) was published by Annual Review of Biophysics.

Posted with permission from the Annual Review of Biophysics, Volume $48^{\circ}$ by Annual Reviews, http://www.annualreviews.org (self-archived preprint:

https:/www.annualreviews.org/page/authors/author-instructions/distributing/self-archiving). 


\section{INTRODUCTION}

Life is based on communication. Even individual cells communicate as they receive messages from the outside of the cell that guide their activity and functions. This information is received at the cell membrane surface from which it is directed to the correct destination inside the cell. If anything in this process goes wrong, the consequences are often unpleasant - in the most severe cases a disease. In essence, communication is crucial for survival.

The information arriving to the cell is primarily recognized by receptors embedded in cell membranes. Given that cell membranes are mostly composed of lipids, a number of intriguing questions emerge about the role of lipids in cellular signaling. How lipids modulate the receptor activation and function? Is it possible that lipids could act as messages, or messengers, such that their transport would also be a means of communication? Is it possible that the function of lipids could be re-programmed by chemical modification?

Quite surprisingly, there are major gaps in our understanding regarding the role of lipids in cellular communication. This largely stems from the tiny scales in space and time that are typical for molecular-scale signaling processes. However, this is exactly the region where molecular simulation techniques are often the method of choice.

Here in this review we discuss how atomistic and coarse-grained molecular simulations can be used to unravel how lipids contribute to cellular signaling. Given the conciseness of this article, the aim of this review is not to be exhaustive - rather we discuss selected examples of recent work where computer simulations have shown the added value that they can provide to complement experiments. Other review articles discussing related topics, largely from an experimental point of view, are available in the literature $(23,98)$.

\section{LIPID FLIP-FLOPS AS PROCESSES DELIVERING INFORMATION ACROSS MEMBRANES}

Lipid translocation across biological membranes (flip-flop) is of profound importance in cell physiology. Phospholipids are mainly synthesized on the cytoplasmic face of the endoplasmic reticulum (ER) and must be scrambled between the two leaflets for uniform expansion of ER during cell growth (98). In plasma membranes, flip-flops are needed to establish and also dissipate the characteristic transmembrane lipid asymmetry, where the asymmetry is crucial for activating blood coagulation and apoptosis $(37,97)$. Specialized transporter enzymes such as ATP-dependent flippases and floppases use metabolic energy to translocate specific lipids across the membrane. Scramblases in turn promote rapid, non-specific, and bidirectional movement of lipids without consuming ATP. It is intriguing that despite decades of active research, the molecular identities of scramblases and the understanding of their function are only beginning to emerge.

The first X-ray crystal structure of a scramblase (TMEM16) was reported in 2014 (12). The homodimeric structure of TMEM16 contains a membrane-faced, transbilayer hydrophilic groove on each of the subunits, through which the polar headgroup of a lipid can slide across the membrane, keeping the acyl chains in a favorable hydrophobic environment in a fashion that is analogous to swiping a credit card through a card reader $(8,12)$ (Figure 1). Meanwhile, earlier Menon et al. discovered unexpected and robust scrambling activity for rhodopsin (a G protein-coupled receptor (GPCR)), which is structurally very different from TMEM16 (60). Upon reconstitution into vesicles, different conformational states of rhodopsin were observed to facilitate rapid scrambling of phospholipids $\left(>10^{4}\right.$ lipids per rhodopsin per second), speeding translocation up by a factor of $\sim 1000$ and implying that conformational changes in the scramblase protein are not required for scrambling (27). Similar scrambling ability for the apo as well as the holo forms suggests that the lipid translocation path does not involve the 
water-containing central core of the protein (27), which was proposed also earlier (60). These results suggest that the transverse diffusion of lipids along the rhodopsin-membrane interface could be a possible scrambling mechanism $(21,27)$ (Figure 1).

Computer simulations can provide precise atom-scale understanding of how the GPCRfacilitated scramblase function takes place. This is highlighted in recent simulation studies. In ref. (71), Nieminen et al. used extensive atomistic molecular dynamics (MD) simulations to provide intriguing insight into the lipid scrambling mechanism of opsin - the apoprotein of rhodopsin. The study assessed the feasibility of various lipid transport routes and showed that opsin provides favorable conditions for the flip-flop of phospholipids along the protein surface. By fostering the hydration of the lipid headgroup as it glided next to the helices II, III, and IV on the opsin surface, the free energy barrier of phospholipid flip-flop was reduced by $\sim 50 \%$ compared to a protein-free membrane (Figure 2). This likely explains the high scrambling rate found in experiments. The study also concluded that the free energy barrier associated with the translocation of phospholipids through the central water core of opsin is very high, thus supporting the prediction previously made based on experiments (60). The simulation results (71) further suggested that the scramblase activity of opsin is not lipidselective, since the study showed opsin to lower the free energy barrier of cholesterol translocation by $\sim 60 \%$ compared to a protein-free membrane with the same lipid composition (Figure 2). Similar conclusions were drawn for another receptor in the class-A GPCR family ( $\beta_{2}$-adrenergic receptor), which was also found to reduce the free energy barrier of cholesterol translocation, in agreement with experiments. Overall, these simulations (71) provide mechanistic insight into how selected proteins in the GPCR family function as ATPindependent non-selective lipid scramblases.

The molecular mechanism by which opsin facilitates rapid phospholipid scrambling has been explored also in another recent simulation study (62). The study reported that translocation took place in a manner where the lipid headgroup migrates along a hydrophilic path between the transmembrane helices 6 and 7 of opsin, while the lipid chains remained in the hydrophobic membrane region - similar to the "credit card model" proposed by experiment. The Markov state model analysis discussed in this study suggests that the rate-limiting step of the scrambling process is the opening of the hydrophilic path triggered by the conformational changes in opsin structure, where the internal hydration of opsin plays a key role in the scrambling mechanism (62).

Recent studies have shown that lipid flip-flops can be facilitated by synthetic transmembrane (TM) peptides characterized by low complexity (47). TM peptides can induce packing defects in the bilayer due to movement of the helices, local bilayer thinning, and transient penetration of water into the bilayer (38). Facilitated by defects at the lipid-helix interface, a lipid may "slip" into the membrane hydrophobic core with its headgroup in a solvated state and then "pop out" on the other side of the membrane (21). A recent study, which combined experiments and MD simulations, showed that the lipid scrambling activity of a single TM model peptide depends on the hydrophobic length of the peptide and the hydrophilicity of its central residues (66). Short peptides were observed to cause membrane thinning under negative mismatch, and the presence of a hydrophilic residue in the membrane core region fostered the penetration of water to the membrane interior. Together, these factors promote peptide-induced phospholipid flip-flop. An example highlighting this in practice is discussed in ref. (90), which demonstrated through atomistic simulations that the transmembrane WALP $_{23}$ peptide facilitates phospholipid flip-flop by lowering the free energy barrier of lipid translocation. 


\section{LIPID TRANSLOCATION MEDIATED BY MEMBRANE DEFECTS}

In defect-free lipid membranes without proteins, passive lipid flip-flop is a very slow process - typical rates per lipid being of the order of 1/day (1). However, this does not mean that flipflops would not take place and the asymmetric lipid distributions in cell membrane structure would last forever even without ATP-driven non-equilibrium transport. Simulations have revealed that the slow rate is due to the energetically costly transport of the polar lipid headgroups through the hydrophobic membrane interior. As demonstrated by simulations, this can be speeded up by defects, such as membrane pores (29). Then the rate-limiting step in translocation is the formation of pores (defects) that can be induced by, e.g., an applied external electric field, transmembrane ion imbalance, or the presence of surface-active molecules or antimicrobial peptides $(6,30,49,99)$. The kinetics and thermodynamics of passive lipid translocation thereby strongly depend on membrane properties, such as lipid acyl chain length, headgroup chemistry, bilayer phase behavior (lipid packing), and the concentration of cholesterol that quite strongly modulates membrane packing $(1,7,17,52,89)$.

\section{PHOSPHATIDYLSERINE AS A MESSENGER OF DEATH - AND THE ROLE OF OXIDATIVE STRESS}

The most prominent loss of transmembrane lipid asymmetry occurs during programmed cell death (apoptosis) - a coordinated process controlling the removal of damaged cells $(4,9,22)$. In healthy cells phosphatidylserine (PS) is confined to the cytoplasmic leaflet of the plasma membrane, while in cells undergoing apoptosis, PS is exposed to the exoplasmic leaflet. PS exposure therefore serves as an "eat-me" signal that ensures effective recognition and engulfment of the dying cell by phagocytes. Given this, PS is a highly effective marker of apoptosis and has clinical applications in, e.g., cancer therapy and infections. Scramblases such as proteins belonging to the TMEM16 and Xk-related families are known to randomize the transmembrane PS distribution, while flippases such as P4-ATPase restore PS to the cytosolic leaflet (65). Intriguingly, despite extensive research, the precise role of these specific enzymes in apoptosis and their differential lipid translocation mechanisms remain obscure.

Another hallmark of apoptosis is oxidative stress. It takes place when the compensatory antioxidant defense of a cell is overwhelmed by excessive production of reactive oxygen species (ROS), which can oxidize membrane lipids and damage cell membranes. Recent studies $(83,100)$ have demonstrated that the oxidative damage of the plasma membrane can effectively catalyze PS scrambling in a non-enzymatic fashion.

Using MD simulations, Volinsky et al. estimated the free energy of POPS (1-palmitoyl-2oleyl-sn-glycero-3-phospho-L-serine) translocation in the presence and absence of oxidized POPC (1-palmitoyl-2-oleoyl-sn-glycero-3-phosphocholine) (100). The oxidized POPC simulated in this study had a truncated $s n-2$ hydrocarbon chain linked to an aldehyde group at the chain end (PoxnoPC), which is one of the major oxidation products in polyunsaturated phosphatidylcholines (PCs). In a membrane with $20 \mathrm{~mol} \%$ of PoxnoPC, one observed a 20 $\mathrm{kJ} / \mathrm{mol}$ reduction in the free energy barrier of PS translocation, which can enhance the flipflop rate by a factor of $\sim 10^{3}-10^{4}$. To validate the simulation predictions, the authors also measured the transmembrane lipid diffusion using fluorescence techniques (100). They observed a profound enhancement of PS flip-flop in liposomes containing oxidized PC: e.g., the half-time was found to be $\sim 2$ hours in membranes with 0.16 mol fraction of PoxnoPC, while in intact membranes the half-time was several weeks. These findings are supported by another simulation study, which showed the free energy barrier of PS translocation to decrease linearly with increasing concentration of peroxidized PC (83) (Figure 3). For 50 
mol\% peroxidized PC, the free energy barrier of POPS translocation was found to drop $\sim 30 \%$ compared to an intact membrane. Interestingly, both of the above-mentioned computational studies indicate that the translocation of PS is accompanied with the formation of a membrane defect, which facilitates the crossing of the polar lipid head group through the hydrophobic core of the membrane. In membranes rich in oxidized lipids, the polar oxidized group most obviously lowers the energy cost of formation of membrane defects/pores, thus reducing the free energy barrier of PS translocation.

PS externalization in response to high-field nanosecond-long electric pulses was demonstrated in ref. (99) using a combination of simulations and fluorescence microscopy imaging. In simulations of asymmetric bilayers (with PS located in one of the leaflets) subjected to a high transmembrane voltage $(>450 \mathrm{mV} / \mathrm{nm})$, one observed fast intrusion of water on the cathode side of the membrane, leading to pore formation. The anionic PS head group was then electrophoretically dragged along the pore wall. The authors showed that PS translocates only after pore formation and always to the anode side of the membrane, in line with the experimental observations made on live cells (99). Similar electroporation and PS externalization were reported earlier based on coarse-grained simulations (35). The fielddriven alignments of water and lipid-head group dipoles are critical for electropermeabilization and anode-directed PS translocation.

\section{POLYUNSATURATED FATTY ACIDS INVOLVED IN STRESS RESPONSE UNDER OXIDATIVE STRESS}

As described above, an excessive amount of reactive oxygen species causes cellular damage and contributes to aging and age-related disorders. The significance of the problem is illustrated by the fact that nearly $5 \%$ of the total energy used by the body is spent for maintaining and repairing cell membranes damaged by oxidation (11). In this context, the primary targets of ROS are polyunsaturated fatty acids (PUFAs) due to the numerous double bonds in their hydrocarbon chains. In phospholipids, two major oxidized lipid products of the PUFA chain in the $s n-2$ position typically include either a hydroperoxide group or a truncated chain containing an aldehyde or a carboxylic group at the terminus (102). When a hydrophilic group is attached to the $s n-2$ chain, the acyl chain is expected to change its orientation in order to contact water ("acyl chain reversal"), unlike intact PUFA chains that adopt a typical orientation towards the hydrophobic core of a bilayer. The reorientation of the acyl chain depends on the chemical structure of the oxidative modification. For example, an oxidized chain with a terminal carboxyl group in the deprotonated state flips towards water along the bilayer normal, whereas the protonated form of the chain adopts a parallel orientation along the lipid-water interfacial plane (59). In the same context, it was found that while the hydroperoxide group of an oxidized lipid rises to the membrane surface, the peroxyl radical intermediate is instead positioned in the innermost region of a membrane like a non-oxidized lipid (24). The acyl chain reversal increases the area per lipid, leads to bilayer thinning, and decreases lipid chain conformational order, thus promoting water permeability and membrane deformation. The effects of oxidized lipids on the structure and permeation properties of lipid bilayers have been studied by simulations $(10,51,68,75,93,101)$ and experiments $(39,87)$ quite extensively.

A recent computational study demonstrated how the polar groups of oxidized lipids perturb the bilayer, induce formation of pores and other membrane defects, and also induce micellation (10). In a binary mixture of polyunsaturated PCs and their peroxide and aldehyde products, one observed formation of membrane defects with both peroxide and aldehyde lipids, but full pores were found only with aldehyde at medium concentrations $(\sim 50 \mathrm{~mol} \%)$. With aldehyde lipids, the hydrophilic tips of the oxidized chains were highly mobile inside 
the bilayer and got into contact with lipids of the opposing leaflet. Consequently, the aldehyde groups pulled water into bilayer interior, leading to the formation of a water pore stabilized by the flipping of lipid headgroups along the pore wall (Figure 4) (10). At higher aldehyde concentrations $(>75 \mathrm{~mol} \%)$, the membrane pores were unstable and the bilayer deformed into a micelle (Figure 4). Meanwhile, no pores or micelles were formed with peroxidized lipids (10), suggesting that the highly polar peroxidized groups prefer the membrane-water interface to the bilayer interior.

\section{CHOLESTEROL AND OXIDATIVE STRESS}

The role of cholesterol in oxidative stress and the related signaling phenomena is twofold. First, results for intact (non-oxidized) cholesterol demonstrate that cholesterol has a protecting effect against mechanical instability arising from lipid oxidation. Recent computational studies showed that cholesterol forms hydrogen bonds with the oxidized lipids and thereby prevents pore formation and micellation (75). The condensing and ordering effects of the sterol backbone were found to be significant even in a massively peroxidized membrane with all phospholipids and cholesterols peroxidized (68).

Second, cholesterol itself can also be oxidized through the double bond in the ring of cholesterol or H-atom transfer from the allylic position (102). ROS generate ring-oxidized sterols, whereas tail-oxidized sterols are produced by enzymatic oxidation. Despite their relatively low concentrations at physiological conditions, oxysterols are primary players in many biological processes including signaling, given that there quite a few oxysterol-binding membrane receptors (40). As shown by MD simulations for a wide range of ring- and tailoxidized sterols, the ordering and condensing effects of oxysterols correlate with their orientation in a lipid bilayer $(42,74)$. While ring-oxidized sterols behave more or less similar to cholesterol, tail-oxidized sterols can orient themselves either parallel or perpendicular to the bilayer normal due to an additional polar group at the hydrocarbon chain. Oxysterols with rather weak headgroup polarity (e.g., the $3 \beta-\mathrm{OH}$ group replaced by the ketone group in 4cholesten-3-one) undergo frequent interleaflet movements or flip-flop motion $(69,86)$. Also, this oxidized derivative has a lower free-energy barrier for desorption from the membrane to the water phase than cholesterol, and it can be readily transferred to its extracellular acceptors (69), which has a direct consequence to the cellular trafficking of cholesterol.

As discussed above, lipid flip-flops in the absence of scramblases are usually very slow, however sterols seem to be an exception to this rule. A variety of simulation studies have determined free energy barriers associated with cholesterol translocation $(7,17,69)$ and identified the flip-flop mechanism, which is based on cholesterol turning around in the middle of the bilayer. For oxysterols, the flip-flop mechanism can be distinctly different as highlighted by a recent computational study, which revealed a novel translocation mechanism for tail-oxidized sterols (41): 27-OH-cholesterol was shown to move back and forth along the membrane normal with either its $3 \beta-\mathrm{OH}$ group (in the head) exposed to water with the $27-\mathrm{OH}$ group (in the tail) buried inside the membrane, or vice-versa. This "bobbing" mechanism does not require the energetically costly change in sterol orientation during the flip-flop process and thereby renders the translocation process extremely fast (41). For a related oxysterol (25-OH-cholesterol) it has been demonstrated that it transfers from the plasma membrane to the endoplasmic reticulum with a rate that is 100 times faster than that of cholesterol $(45,46)$. For $24 \mathrm{~S}-\mathrm{OH}$-cholesterol and 27-OH-cholesterol the transport from erythrocytes to the plasma membrane is fast, while the transport of cholesterol is almost negligible (58). While the reasons for the distinctly different transport rates are not known, it is quite possible that the rapid translocation in terms of the bobbing mechanism plays a role in the rapid signaling mediated by selected oxysterols. 


\section{MEMBRANE REGISTRATION AND INTERDIGITATION - MEANS FOR CROSS- TALK BETWEEN BILAYER LEAFLETS}

Plasma membranes of eukaryotic cells are characterized by considerable transbilayer lipid asymmetry. Sphingomyelin (SM) is more concentrated in the extracellular leaflet, whereas phosphatidylserine (PS) and phosphatidylethanolamine (PE) are more confined to the cytosolic leaflet $(37,98)$. The distribution of cholesterol is a matter of debate, but it is inferred to be concentrated more in the extracellular leaflet by virtue of its predicted association with SM (37). If the two leaflets were uncoupled, then only the extracellular leaflet would be expected to form raft-like domains. Such transbilayer compositional asymmetry may lead to phase asymmetry or antiregistration, meaning that the two opposing leaflets have different fluidity. Alternatively, a domain in one leaflet can induce the formation of a similar domain in the opposite leaflet, leading to phase symmetry or registration $(57,70)$. This paradigm may explain why certain proteins that are exclusively associated with the cytosolic leaflet of the plasma membrane are co-localized with raft components (103). But then, how do the two leaflets of a biological membrane modulate each other's physical properties? While several plausible mechanisms have been proposed $(18,57)$, the idea of dynamic chain interdigitation is one of the most promising ways to understand the crosstalk between the membrane leaflets.

A recent study employed atomistic simulations to assess how the extracellular and cytosolic leaflets of membranes interact through interdigitation (85). The study used realistic model membrane systems, which closely mimicked the composition of eukaryotic plasma membranes. The results revealed that SM with a long saturated acyl chain (SM 18:1-24:0) protruded deep into the opposing bilayer leaflet, and the penetration was particularly strongly when the bilayer was asymmetric. The interdigitation affected the conformational order of the penetrating SM acyl chain as well as the order of the opposing membrane leaflet, thereby strengthening the interaction and the coupling across the membrane (85). A similar mechanism was reported in another atom-scale simulation study, where a long-chain ganglioside (GM1) was observed to promote transbilayer coupling through interdigitation (53). To unravel how the acyl chain length of GM1 contributes to membrane registration, the study also used coarse-grained simulations probing longer timescales in large membrane systems. The results showed that with short-chain GM1 lipids (with 18 carbons) in the extracellular leaflet, both bilayer leaflets readily underwent complete phase separation leading to a phase-symmetric bilayer with strong membrane registration (Figure 5). In contrast, GM1 with an extended acyl chain ( $\sim 30$ carbons) was found to perturb the phase of the GM1-free cytosolic leaflet. The significant interdigitation of the long acyl chain of GM1 into the opposite leaflet was observed to induce the mixing of saturated and unsaturated lipids, thus preventing or at least slowing down the phase separation in the cytosolic leaflet and weakening the overall membrane registration process (Figure 5) (53).

An earlier study using extensive coarse-grained simulations showed a transition from the registered to antiregistered bilayer configurations, when the length of the saturated lipids was increased from $\sim 16$ to $\sim 20$ carbons, increasing the height mismatch between ordered and disordered domains by $\sim 0.4 \mathrm{~nm}(80)$. The results showed that in compositionally symmetric phase-separated bilayers composed of cholesterol, saturated, and unsaturated lipids, the transbilayer phase asymmetry results from the mismatched acyl chain lengths. Theoretical models have suggested that domain registration is directed by the balance between two tensions (57): the interleaflet tension at the bilayer midplane, which is minimized by transbilayer registration, and the intra-leaflet tension at domain boundaries due to height mismatch, which can be relieved by mixing saturated and unsaturated lipids at domain boundaries (80). 
Concluding, the simulation studies suggest that the physical interactions between the two leaflets in the form of interdigitation provide a means to mediate transmembrane communication associated with signal transduction.

\section{LIPID MESSENGERS BIND TO RECEPTORS AND TARGET PROTEINS AS ORTHOSTERIC LIGANDS}

Lipids are not just passive constituents of cell membranes or a source of stored energy; they can also act as signaling molecules (23). Extracellular stimuli or "primary messengers" can elicit cellular responses through generation of intracellular "second messengers". Agonists of cellular receptors, such as GPCRs and tyrosine kinases, activate enzymes such as phospholipases, sphingomyelinases, and phosphatidylinositol-3-kinase, which catalyze the cleavage of lipids (73). Lipid metabolites such as sphingosine-1-phosphate (S1P), diacylglycerol (DAG), or ceremide are important examples of lipid messengers, which serve as intracellular signals and bind target proteins to mediate specific cellular responses.

The mechanisms of lipid signal transduction are poorly understood, but often involve GPCRs. As reviewed recently (96), over 50 GPCRs have been implicated in signaling lipids that include lysophospholipids, phospholipids, fatty acids, and eicosanoids. Dysregulation of these lipid GPCRs contribute to diverse cancer-related processes and may be therapeutically exploited (96). Most lipid GPCRs belong to class-A (Rhodopsin-like) GPCRs. However, unlike prototypical GPCRs that recognize small hydrophilic ligands, lipid GPCRs are activated by lipid mediators, which possess a polar head group and long hydrophobic moieties, thus implying significantly different activation mechanisms. Sphingosine-1-phosphate receptors $\left(\mathrm{S}_{\mathrm{PR}} \mathrm{PR}_{1-5}\right)$ and lysophosphatidic acid receptors belong to this receptor superfamily. In the past few years, crystal structures of several lipid GPCRs have been resolved, including the structure of antagonist-bound S1PR 1 (33). S1P activates five cell surface GPCRs $\left(\mathrm{SIPR}_{1-5}\right)$. By combining MD simulations and functional assays using S1P analogs with different alkyl chain lengths, a recent study demonstrated that the alkyl chain length of the lipidic agonist is the key structural feature in the activation of S1P receptors (95). The data suggest that the headgroup of S1P is required for high-affinity binding, but the alkyl chain is responsible for triggering the activation. S1P stabilizes the active state of $\mathrm{S} 1 \mathrm{PR}_{1}$ by inserting its lipid chain into the small hydrophobic cavity of the receptor between TM domains 3, 5, and 6. The ligand efficacy was observed to be directly related to the alkyl chain length and to vary within receptor subtypes due to different volumes of binding cavities (95). Another recent work by the McCammon group investigated the activation mechanism of ligand-free $\mathrm{S}_{1} \mathrm{PR}_{1}(15)$. The simulations captured four independent activation events and correlated helix movement during activation. During the simulations, a lipid molecule was repeatedly found to enter the receptor between the extracellular ends of TM1 and TM7.

Cholesterol is known for its modulatory role on GPCRs. Cholesterol and its derivatives are even speculated to modulate functions of certain class-A GPCRs from the orthosteric binding pocket, similar to conventional class-A ligands $(5,92)$. A recent crystal structure shows a cholesterol molecule at the binding site of the extracellular domain of a class-F GPCR (14). The rather surprising orthosteric mode of action of cholesterol is less explored. It has been mostly postulated that cholesterol can allosterically regulate GPCRs by directly interacting with them at specific sites on the receptor surface or by indirectly changing the physical properties of the membrane $(25,76)$. In certain cases, however, the impact of cholesterol on GPCRs can go beyond pure allosteric modulation. By combining experimental and computational approaches, a recent study showed that membrane cholesterol can spontaneously invade into the orthosteric ligand binding pocket of adenosine $\mathrm{A}_{2 \mathrm{~A}}$ receptor 
$\left(\mathrm{A}_{2 \mathrm{~A}} \mathrm{R}\right)$ (Figure 6) and compete with specific ligand binding (28). In addition to several interaction sites on the receptor surface, simulations demonstrated high cholesterol densities to populate a significant area of the orthosteric ligand-binding site of the receptor, overlapping with the position of the ligand found in the crystal structure (Figure 6). Cholesterol entered the binding pocket through the extracellular side of TM5-6 using the same portal gate that was previously suggested for the entry of a ligand into opsin. Confirming the simulations, experiments showed specific ligand binding to increase for decreasing cholesterol content. Further, the authors confirmed the presence of cholesterol inside the receptor by chemical modification of the receptor interior in a biotinylation assay. The study strongly suggests a new regulatory mechanism of cholesterol and also provides an example of how simulations can bring added value to experiments (28).

\section{LIPIDS IN ALLOSTERIC MODULATION OF RECEPTOR ACTIVATION}

Lipids are progressively emerging as essential modulators of membrane protein functions, including major families of cellular receptors such as GPCRs $(19,25,82)$, receptor tyrosine kinases $(36,61)$, T-cell receptors $(94)$, and ligand/voltage gated ion channels $(3,31,43,78)$. These receptors are signaling machines embedded in cell membranes, which convert extracellular stimuli into cellular responses. Biological membranes host dynamic lipid-protein assemblies, where a protein with selected lipids may create its own functional lipid microenvironment $(44,78)$. Several studies have identified and characterized lipid interaction sites in a variety of membrane proteins $(34,44,78)$. A lipid specifically bound to a protein at a site that is not an orthosteric site may also regulate protein structure and function, a process referred to as allosteric modulation. Despite substantial instances of lipid modulation, the mechanisms of allosteric modulation remain unclear. MD simulations could offer detailed insight into specific lipid-protein interactions and their effects $(2,91)$, but so far only limited computational studies have been able to link these physical interactions to functional consequences of receptor activation/function. These studies have mostly focused on GPCRs due to their involvement in a multitude of physical processes, the acknowledged lipiddependency of GPCRs, and also the availability of several crystal structures, which make GPCRs viable for computational investigations.

Biologically relevant phospholipids can act as allosteric regulators of GPCR function. A recent experimental study showed that lipids with the phosphatidylglycerol (PG) headgroup strongly favor agonist binding and receptor activation of $\beta_{2}$-adrenergic receptor $\left(\beta_{2} \mathrm{AR}\right)$, whereas the PE headgroup favors antagonist binding and stabilizes the inactive state of the receptor (19). A recent computational study provided mechanistic insight into the positive and negative allosteric modulation and showed that the effect is attributed to the chemical differences and charges of these lipid headgroups, which modify the lipid-receptor interactions and alter membrane properties (13). Simulations also showed that the negatively charged 1,2-dioleoyl-sn-glycero-3-phosphoglycerol (DOPG) lipid preferentially interacts with the positively charged residues of the intercellular loop 3 and the intercellular end of TM6, which give rise to an outward pull on TM6 and stabilize receptor conformation with an open $G$ protein binding interface that is characteristic to the active state of the receptor. Further, this study, and also an earlier computational investigation, showed that PG from the cytosolic leaflet intrudes into the empty G protein-binding site between TM6 and TM7 of $\beta_{2} \mathrm{AR}$ that is in the active state $(13,67)$. The negatively charged PG headgroup specifically interacts with R131 $1^{3.50}$ on TM3 and inhibits the formation of a conserved R131 $1^{3.50}-\mathrm{E} 268^{6.30}$ ionic lock, which characterizes the inactive state of the receptor. These studies suggest that the binding of anionic PG stabilizes the active state of the receptor via conformational selection. Meanwhile, the neutral 1,2-dioleoyl-sn-glycero-3-phospho-ethanolamine (DOPE) 
lipids (with a positively charged ethanol amine moiety in the headgroup) create unfavorable interactions with the positively charged residues of TM6, thereby destabilizing the active state and strongly favoring complete deactivation of $\beta_{2} \mathrm{AR}$ (13).

Another recent computational study showed that the PE headgroup and the polyunsaturated docosahexaenoic acid chains preferentially bind to the inactive conformation of rhodopsin, suggesting that the lipid modulation arises from both solvent-like (membrane mediated) and ligand-like (direct lipid-protein) interactions that depend on the state/conformation of the receptor (88). Ref. (13) further showed that another neutral lipid (1,2-dioleoyl-sn-glycero-3phosphocholine (DOPC)) can induce partial deactivation of $\beta_{2} \mathrm{AR}$, in agreement with computational results by Dror et al. that showed gradual inactivation of $\beta_{2} \mathrm{AR}$ in a POPC membrane $(20,48)$. Unlike $\mathrm{PE}$, the $\mathrm{PC}$ headgroup is more hydrophobic and unable to form inter-lipid hydrogen bonds, resulting in a lower membrane density. In a less dense DOPC membrane, $\beta_{2} \mathrm{AR}$ exhibits greater conformational freedom and inactivates slower than in a DOPE bilayer, in agreement with experimental results (19).

Cholesterol is frequently invoked as a modulator of protein structure $(16,55,64)$, stability $(32,104)$, oligomerization (reviewed in ref. (79)), and ligand-binding of several GPCRs $(25,26,72,76,77,81,82)$. The literature reporting the functional role of cholesterol as a positive or negative modulator of GPCR activity is extensive. It has been a matter of intense debate whether the modulation is due to cholesterol-induced changes in membrane properties, specific and direct cholesterol-GPCR interactions, or both. Crystal structures have revealed the binding of cholesterol to the surface of several GPCRs $(25,32,34)$, suggesting specific allosteric sites for cholesterol. However, the functional relevance of these physical interactions and the atomic-scale mechanism of cholesterol modulation have remained unknown. A recent computational study clarified this issue to a large degree, however. It provided atomistic insight into the mechanism of allosteric regulation of $\beta_{2} \mathrm{AR}$ by cholesterol (56). The study showed that under cholesterol-rich conditions, cholesterol has a large impact on the conformational dynamics of $\beta_{2} \mathrm{AR}$, as cholesterol limits the receptor conformation predominantly to one state. Under cholesterol-poor conditions, the conformational distribution was found to be very broad $(54,56)$. The mechanism of action was revealed to be based on binding of cholesterol at two specific interaction sites located near the TM5-6-7 domains, which are evolutionally conserved among $\beta_{2} \mathrm{AR}$ orthologues. Cholesterols bound at these sites significantly impeded the mobility of the respective helices, thereby reducing the overall conformational flexibility of the receptor. The effect was found to depend on the strength of cholesterol binding and not on the physical state of the surrounding bilayer (56). The study also showed that cholesterol bound between TM5-TM6 on the intracellular side exerts the strongest effect by preventing the outward movement of TM6 required for activation of $\beta_{2} \mathrm{AR}$. On the other hand, cholesterol also stabilizes the active state of the receptor by preventing the inward movement of TM6. Altogether, the study shows that cholesterol reduces the conformational flexibility of $\beta_{2} \mathrm{AR}$, weakening chances to switch between different functional states (56).

\section{LIPIDS MODULATING LIPID RECEPTORS}

Not all receptors are proteins. There are also quite a few lipids acting as receptors, glycosphingolipids being perhaps the primary example of this class. Glycolipids are challenging to consider through computer simulations, since in these lipids the carbohydrate headgroup carries out the recognition that is highly dependent on the chemical details of the headgroup. Instead of coarse-grained models, the most appropriate approach is to use atomistic simulations. Lingwood et al. (50) used this strategy to reveal the mechanism used by cholesterol to modulate the receptor function of GM1. By inducing a tilt in the GM1 
headgroup, cholesterol was able to induce a loss of access for ligand binding. Recent simulations by Rissanen et al. (84) support this picture. Exploring this issue more broadly would be exceptionally justified.

\section{CONCLUDING REMARKS}

Cell signaling coordinates cell actions and controls a variety of cellular processes. Errors in signaling often lead to diseases such as cancer and diabetes. By understanding the underlying physical and chemical bases of signaling, the chances of clarifying why and how these diseases emerge would be significantly improved. Even though membrane proteins are the main contributor to cellular communication taking place in or through cell membranes, there is reason to keep in mind that membrane proteins are modulated by a number of factors, in particular lipids. In this article, we have discussed how lipids contribute to and take part in cell signaling. Among many other ways, lipids modulate protein conformations, act as messengers, and they are subject to structural modifications that can change the lipids' functions in the context of signaling. Further, even though the space is not enough to discuss this topic more broadly, it is worth to mention that lipids can also control protein activation by, e.g., modulating the partitioning of membrane proteins by varying membrane physical properties, and by altering the intramembrane pressure profile exerted on transmembrane proteins. There are many ways, but the very essence of the matter remains the same: the role of lipids in cellular signaling is significant. And - the added value given by simulations is impressive.

\section{ACKNOWLEDGEMENTS}

We thank the Department of Biotechnology, Government of India, for financial support under Bio-CARe 2016 program for Women Scientists (No. BT/PR17981/BIC/101/576/2016) (MM). We also acknowledge financial support granted by the Sigrid Juselius Foundation, HiLIFE (University of Helsinki), Academy of Finland (Centre of Excellence project (grant no. 307415)), and the European Research Council (Advanced Grant project CROWDEDPRO-LIPIDS (grant no. 290974)). CSC — IT Center for Science (Espoo, Finland) is acknowledged for computing resources. 


\section{REFERENCES}

1. Allhusen JS, Conboy JC. 2016. The ins and outs of lipid flip-flop. Acc. Chem. Res. 50:58-65.

2. Aponte-Santamaría C, Briones R, Schenk AD, Walz T, de Groot BL. 2012. Molecular driving forces defining lipid positions around aquaporin-0. Proc. Natl. Acad. Sci. USA 109:9887-9892.

3. Baenziger JE, Morris ML, Darsaut TE, Ryan SE. 2000. Effect of membrane lipid composition on the conformational equilibria of the nicotinic acetylcholine receptor. J. Biol. Chem. 275:777-784.

4. Balasubramanian K, Schroit AJ. 2003. Amino phospholipid asymmetry: a matter of life and death. Annu. Rev. Physiol. 65:701-734.

5. Benned-Jensen T, Norn C, Laurent S, Madsen CM, Larsen HM, Arfelt KN, Wolf RM, Frimurer T, Sailer AW, Rosenkilde MM. 2012. Molecular characterization of oxysterol binding to the Epstein-Barr virus-induced gene 2 (GPR183). J. Biol. Chem. 287:35470-35483.

6. Bennett WD, Hong CK, Wang Y, Tieleman DP. 2016. Antimicrobial peptide simulations and the influence of force field on the free energy for pore formation in lipid bilayers. J. Chem. Theory Comput. 12:4524-4533.

7. Bennett WD, MacCallum JL, Hinner MJ, Marrink SJ, Tieleman DP. 2009. Molecular view of cholesterol flip-flop and chemical potential in different membrane environments. J. Am. Chem. Soc. 131:12714-12720.

8. Bethel NP, Grabe M. 2016. Atomistic insight into lipid translocation by a TMEM16 scramblase. Proc. Natl. Acad. Sci. USA 113:14049-14054.

9. Bevers EM, Williamson PL. 2016. Getting to the outer leaflet: physiology of phosphatidylserine exposure at the plasma membrane. Physiol. Rev. 96:605-645.

10. Boonnoy P, Jarerattanachat V, Karttunen M, Wong-ekkabut J. 2015. Bilayer deformation, pores, and micellation induced by oxidized lipids. J. Phys. Chem. Lett. 6:48844-4848.

11. Brenna JT, Carlson SE. 2014. Docosahexaenoic acid and human brain development: evidence that a dietary supply is needed for optimal development. $J$. Hum. Evol. 77:99-106.

12. Brunner JD, Lim NK, Schenck S, Duerst A, Dutzler R. 2014. X-ray structure of a calcium-activated TMEM16 lipid scramblase. Nature 516:207.

13. Bruzzese A, Gil C, Dalton JA, Giraldo J. 2018. Structural insights into positive and negative allosteric regulation of a $\mathrm{G}$ protein-coupled receptor through protein-lipid interactions. Sci. Rep. 8:4456.

14. Byrne EF, Sircar R, Miller PS, Hedger G, Luchetti G, Nachtergaele S, Tully MD, Mydock-McGrane L, Covey DF, Rambo RP, Sansom MSP, Newstead S, Rohatgi R, Siebold C. 2016. Structural basis of Smoothened regulation by its extracellular domains. Nature 535:517-522.

15. Caliman AD, Miao Y, McCammon JA. 2017. Activation mechanisms of the first sphingosine-1-phosphate receptor. Protein Sci. 26:1150-1160.

16. Casiraghi M, Damian M, Lescop E, Point E, Moncoq K, Morellet N, Levy D, Marie J, Guittet E, Banères JL, Catoire LJ. 2016. Functional modulation of a G protein-coupled receptor conformational landscape in a lipid bilayer. J. Am. Chem. Soc. 138:11170-11175.

17. Choubey A, Kalia RK, Malmstadt N, Nakano A, Vashishta P. 2013. Cholesterol translocation in a phospholipid membrane. Biophys. J. 104:2429-2436. 
18. Collins MD, Keller SL. 2008. Tuning lipid mixtures to induce or suppress domain formation across leaflets of unsupported asymmetric bilayers. Proc. Natl. Acad. Sci. USA 105:124-128.

19. Dawaliby R, Trubbia C, Delporte C, Masureel M, van Antwerpen P, Kobilka BK, Govaerts C. 2016. Allosteric regulation of G protein-coupled receptor activity by phospholipids. Nat. Chem. Biol. 12:35-39.

20. Dror RO, Arlow DH, Maragakis P, Mildorf TJ, Pan AC, Xu H, Borhani DW, Shaw DE. 2011. Activation mechanism of the 32 -adrenergic receptor. Proc. Natl. Acad. Sci. USA 108:18684-18689.

21. Ernst OP, Menon AK. 2015. Phospholipid scrambling by rhodopsin. Photochem. Photobiol. Sci. 14:1922-31.

22. Fadeel B, Xue D. 2009. The ins and outs of phospholipid asymmetry in the plasma membrane: roles in health and disease. Crit. Rev. Biochem. Mol. Biol. 44:264-277.

23. Fernandis AZ, Wenk MR. 2007. Membrane lipids as signaling molecules. Curr. Opin. Lipidol. 18:121-128.

24. Garrec J, Monari A, Assfeld X, Mir LM, Tarek M. 2014. Lipid peroxidation in membranes: the peroxyl radical does not "float". J. Phys. Chem. Lett. 5:1653-8.

25. Gimpl G. Interaction of G protein coupled receptors and cholesterol. 2016. Chem. Phys. Lipids 199:61-73.

26. Gimpl G, Burger K, Fahrenholz F. 1997. Cholesterol as modulator of receptor function. Biochemistry 36:10959-10974.

27. Goren MA, Morizumi T, Menon I, Joseph JS, Dittman JS. 2014. Constitutive phospholipid scramblase activity of a $\mathrm{G}$ protein-coupled receptor. Nat. Commun. 5:5115.

28. Guixà-González R, Albasanz JL, Rodriguez-Espigares I, Pastor M, Sanz F, MartíSolano M, Manna M, Martinez-Seara H, Hildebrand PW, Martín M, Selent J. 2017. Membrane cholesterol access into a G-protein-coupled receptor. Nat. Commun. 8:14505.

29. Gurtovenko AA, Anwar J, Vattulainen I. 2010. Defect-mediated trafficking across cell membranes: insights from in silico modeling. Chem. Rev. 110:6077-6103.

30. Gurtovenko AA, Vattulainen I. 2007. Molecular mechanism for lipid flip-flops. $J$. Phys. Chem. B 111:13554-13559.

31. Hansen SB, Tao X, MacKinnon R. 2011. Structural basis of PIP 2 activation of the classical inward rectifier K+ channel Kir2. 2. Nature 477:495-498.

32. Hanson MA, Cherezov V, Griffith MT, Roth CB, Jaakola VP, Chien EY, Velasquez J, Kuhn P, Stevens RC. 2008. A specific cholesterol binding site is established by the $2.8 \AA$ structure of the human $\beta 2$-adrenergic receptor. Structure 16:897-905.

33. Hanson MA, Roth CB, Jo E, Griffith MT, Scott FL, Reinhart G, Desale H, Clemons B, Cahalan SM, Schuerer SC, Sanna MG, Han GW, Kuhn P, Rosen H, Stevens RC. 2012. Crystal structure of a lipid G protein-coupled receptor. Science 335:851-855.

34. Hedger G, Sansom MS. 2016. Lipid interaction sites on channels, transporters and receptors: recent insights from molecular dynamics simulations. Biochem. Biophys. Acta 1858:2390-2400.

35. $\mathrm{Hu} \mathrm{Q}$, Joshi RP, Schoenbach KH. 2005. Simulations of nanopore formation and phosphatidylserine externalization in lipid membranes subjected to a highintensity, ultrashort electric pulse. Phys. Rev. E 72:031902. 
36. Kaszuba K, Grzybek M, Orłowski A, Danne R, Róg T, Simons K, Coskun Ü, Vattulainen I. 2015. N-Glycosylation as determinant of epidermal growth factor receptor conformation in membranes. Proc. Natl. Acad. Sci. USA 112:4334-4339.

37. Kobayashi T, Menon AK. 2018. Transbilayer lipid asymmetry. Curr. Biol. 28:R386-R391.

38. Kol MA, van Laak AN, Rijkers DT, Killian JA, de Kroon AI, de Kuijff B. 2003. Phospholipid flop induced by transmembrane peptides in model membranes is modulated by lipid composition. Biochemistry 42:231-7.

39. Knobloch JJ, Nelson AR, Köper I, James M, McGillivray DJ. 2015. Oxidative damage to biomimetic membrane systems: in situ Fe (II)/ascorbate initiated oxidation and incorporation of synthetic pxidized Phospholipids. Langmuir 31:12679-12687.

40. Kulig W, Cwiklik L, Jurkiewicz P, Rog T, Vattulainen I. 2016. Cholesterol oxidation products and their biological importance. Chem. Phys. Lipids 199:144160.

41. Kulig W, Mikkolainen H, Olżyńska A, Jurkiewicz P, Cwiklik L, Hof M, Vattulainen I, Jungwirth P, Rog T. 2018. Bobbing of oxysterols: molecular mechanism for translocation of tail-oxidized sterols through biological membranes. J. Phys. Chem. Lett. 9:1118-1123.

42. Kulig W, Olżyńska A, Jurkiewicz P, Kantola AM, Komulainen S, Manna M, Pourmousa M, Vazdar M, Cwiklik L, Rog T, Khelashvili G, Harries D, Telkki VV, Hof M, Vattulainen I, Jungwirth P. 2015. Cholesterol under oxidative stress-how lipid membranes sense oxidation as cholesterol is being replaced by oxysterols. Free Radic. Biol. Med. 84:30-41.

43. Laganowsky A, Reading E, Allison TM, Ulmschneider MB, Degiacomi MT, Baldwin AJ, Robinson CV. 2014. Membrane proteins bind lipids selectively to modulate their structure and function. Nature 510:172-175.

44. Landreh M, Marty MT, Gault J, Robinson CV. 2016. A sliding selectivity scale for lipid binding to membrane proteins. Curr. Opin. Struct. Biol. 39:54-60.

45. Lange Y, Ye J, Rigney M, Steck T L. 1999. Regulation of endoplasmic reticulum cholesterol by plasma membrane cholesterol. J. Lipid Res. 40:2264-2270.

46. Lange Y, Ye J, Strebel F. 1995. Movement of 25-hydroxycholesterol from the plasma membrane to the rough endoplasmic reticulum in cultured hepatoma cells. J. Lipid Res. 36:1092-1097.

47. Langer M, Sah R, Veser A, Gütlich M, Langosch D. 2013. Structural properties of model phosphatidylcholineflippases. Chem. Biol. 20:63-72.

48. Latorraca NR, Venkatakrishnan AJ, Dror RO. 2016. GPCR dynamics: structures in motion. Chem. Rev. 117:139-155.

49. Leontiadou H, Mark AE, Marrink SJ. 2006. Antimicrobial peptides in action. $J$. Am. Chem. Soc. 128:12156-12161.

50. Lingwood D, Binnington B, Rog T, Vattulainen I, Grzybek M, Coskun U, Lingwood CA, Simons K. 2011. Cholesterol modulates glycolipid conformation and receptor activity. Nat. Chem. Biol. 7:260-2.

51. Lis M, Wizert A, Przybylo M, Langner M, Swiatek J, Jungwirth P, Cwiklik L. 2011. The effect of lipid oxidation on the water permeability of phospholipids bilayers. Phys. Chem. Chem. Phys. 13:17555-17563.

52. Liu J, Brown KL, Conboy JC. 2013. The effect of cholesterol on the intrinsic rate of lipid flip-flop as measured by sum-frequency vibrational spectroscopy. Faraday Disc. 161:45-61. 
53. Manna M, Javanainen M, Monne HM, Gabius HJ, Rog T, Vattulainen I. 2017. Long-chain GM1 gangliosides alter transmembrane domain registration through interdigitation. Biochim. Biophys. Acta 1859:870-878.

54. Manna M, Kulig W, Javanainen M, Tynkkynen J, Hensen U, Müller DJ, Rog T, Vattulainen I. 2015. How to minimize artifacts in atomistic simulations of membrane proteins, whose crystal structure is heavily engineered: $\beta 2$-adrenergic receptor in the spotlight. J. Chem. Theory Comput. 11:3432-3445.

55. Manna M, Mukhopadhyay C. 2011. Cholesterol driven alteration of the conformation and dynamics of phospholamban in model membranes. Phys. Chem. Chem. Phys. 13:20188-20198.

56. Manna M, Niemelä M, Tynkkynen J, Javanainen M, Kulig W, Müller DJ, Rog T, Vattulainen I. 2016. Mechanism of allosteric regulation of $\beta 2$-adrenergic receptor by cholesterol. eLife 5: e18432.

57. May S. 2009. Trans-monolayer coupling of fluid domains in lipid bilayers. Soft Matter 5:3148-3156.

58. Meaney S, Bodin K, Diczfalusy U, Bjorkhem I. 2002. On the rate of translocation in vitro and kinetics in vivo of the major oxysterols in human circulation: critical importance of the position of the oxygen function. J. Lipid Res. 43:2130-2135.

59. Mendes Ferreira T, Sood R, Bärenwald R, Carlström G, Topgaard D, Saalwächter K, Kinnunen PK, Ollila OH. 2016. Acyl chain disorder and azelaoyl orientation in lipid membranes containing oxidized lipids. Langmuir 32:6524-6533.

60. Menon I, Huber T, Sanyal S, Banerjee S, Barré P, Canis S, Warren JD, Hwa J, Sakmar TP, Menon AK. 2011. Opsin is a phospholipid flippase. Curr. Biol. 21:149-153.

61. Michailidis IE, Rusinova R, Georgakopoulos A, Chen Y, Iyengar R, Robakis NK, Logothetis DE, Baki L. 2011. Phosphatidylinositol-4, 5-bisphosphate regulates epidermal growth factor receptor activation. Pflügers Arch. 461:387-397.

62. Morra G, Razavi AM, Pandey K, Weinstein H, Menon AK, Khelashvili G. 2018. Mechanisms of lipid scrambling by the G protein-coupled receptor opsin. Structure 26:356-367.

63. Muller CP, Reichel M, Muhle C, Rhein C, Gulbins E, Komhuber J. 2015. Biochim. Biophys. Acta 1851:1051-1065.

64. Muth S, Fries A, Gimpl G. 2011. Cholesterol-induced conformational changes in the oxytocin receptor. Biochem. J. 437:541-553.

65. Nagata S, Suzuki J, Segawa K, Fujii T. 2016. Exposure of phosphatidylserine on the cell surface. Cell Death Differ. 23:952-961.

66. Nakao H, Hayashi C, Ikeda K, Saito H, Nagao H, Nakano M. 2018. Effect of hydrophilic residues and hydrophobic length on flip-flop promotion by transmembrane peptides. J. Phys. Chem. B 122: 4318-4324.

67. Neale C, Herce HD, Pomès R, García AE. 2015. Can specific protein-lipid interactions stabilize an active state of the beta 2 adrenergic receptor? Biophys. $J$. 109:1652-1662.

68. Neto AJ, Cordeiro RM. 2016. Molecular simulations of the effects of phospholipid and cholesterol peroxidation on lipid membrane properties. Biochim. Biophys. Acta 1858:2191-2198.

69. Neuvonen M, Manna M, Mokkila S, Javanainen M, Rog T, Liu Z, Bittman R, Vattulainen I, Ikonen E. 2014. Enzymatic oxidation of cholesterol: properties and functional effects of cholestenone in cell membranes. PLoS One 9:e103743.

70. Nickels JD, Smith JC, Cheng X. 2015. Lateral organization, bilayer asymmetry, and inter-leaflet coupling of biological membranes. Chem. Phys. Lipids 192:87-99. 
71. Nieminen T, Manna M, Rog T, Vattulainen I. 2018. Mechanism of non-selective lipid scrambling fostered by GPCRs - atomistic perspective. Manuscript under review.

72. Oates J, Watts A. 2011. Uncovering the intimate relationship between lipids, cholesterol and GPCR activation. Curr. Opin. Struct. Biol. 21:802-807.

73. Okazaki Y, Saito K. 2014. Roles of lipids as signaling molecules and mitigators during stress response in plants. Plant J. 79:584-596.

74. Olsen BN, Schlesinger PH, Baker NA. 2009. Perturbations of membrane structure by cholesterol and cholesterol derivatives are determined by sterol orientation. $J$. Am. Chem. Soc.131:4854-4865.

75. Owen MC, Kulig W, Rog T, Vattulainen I, Strodel B. 2018. Cholesterol protects the oxidized lipid bilayer from water injury: an all-atom molecular dynamics study. J. Membr. Biol. 17:1-4.

76. Paila YD, Chattopadhyay A. 2009. The function of G-protein coupled receptors and membrane cholesterol: specific or general interaction? Glycoconj. J. 26:711720.

77. Paila YD, Jindal E, Goswami SK, Chattopadhyay A. 2011. Cholesterol depletion enhances adrenergic signaling in cardiac myocytes. Biochem. Biophys. Acta 1808:461-465.

78. Patrick JW, Boone CD, Liu W, Conover GM, Liu Y, Cong X, Laganowsky A. 2018. Allostery revealed within lipid binding events to membrane proteins. Proc. Natl. Acad. Sci. USA 115:2976-2981.

79. Periole X. 2016. Interplay of G protein-coupled receptors with the membrane: insights from supra-atomic coarse grain molecular dynamics simulations. Chem. Rev. 117:156-185.

80. Perlmutter JD, Sachs JN. 2011. Interleaflet interaction and asymmetry in phase separated lipid bilayers: molecular dynamics simulations. J. Am. Chem. Soc. 133:6563-6577.

81. Pontier SM, Percherancier Y, Galandrin S, Breit A, Galés C, Bouvier M. 2008. Cholesterol-dependent separation of the beta2-adrenergic receptor from its partners determines signaling efficacy: insight into nanoscale organization of signal transduction. J. Biol. Chem. 283:24659-24672.

82. Pucadyil TJ, Chattopadhyay A. 2006. Role of cholesterol in the function and organization of G-protein coupled receptors. Prog. Lipid Res. 45:295-333.

83. Razzokov J, Yusupov M, Vanuytsel S, Neyts EC, Bogaerts A. 2017. Phosphatidylserine flip-flop induced by oxidation of the plasma membrane: a better insight by atomic scale modeling. Plasma Processes Polym. 14:e1700013.

84. Rissanen S, Grzybek M, Orlowski A, Rog T, Cramariuc O, Levental I, Eggeling C, Sezgin E, Vattulainen I. 2017. Phase partitioning of GM1 and its Bodipy-labeled analog determine their different binding to Cholera Toxin. Front. Physiol. 8:252.

85. Róg T, Orłowski A, Llorente A, Skotland T, Sylvänne T, Kauhanen D, Ekroos K, Sandvig K, Vattulainen I. 2016. Interdigitation of long-chain sphingomyelin induces coupling of membrane leaflets in a cholesterol dependent manner. Biochim. Biophys. Acta 1858:281-288.

86. Róg T, Stimson LM, Pasenkiewicz-Gierula M, Vattulainen I, Karttunen M. 2008. Replacing the cholesterol hydroxyl group with the ketone group facilitates sterol flip-flop and promotes membrane fluidity. J. Phys. Chem. B 112:1946-1952.

87. Runas KA, Malmstadt N. 2015. Low levels of lipid oxidation radically increase the passive permeability of lipid bilayers. Soft Matter 11:499-505. 
88. Salas-Estrada LA, Leioatts N, Romo TD, Grossfield A. 2018. Lipids alter rhodopsin function via ligand-like and solvent-like interactions. Biophys. J. 114:355-367.

89. Sapay N, Bennett WD, Tieleman DP. 2009. Thermodynamics of flip-flop and desorption for a systematic series of phosphatidylcholine lipids. Soft Matter 5:3295-3302.

90. Sapay N, Bennett WD, Tieleman DP. 2010. Molecular simulations of lipid flip-flop in the presence of model transmembrane helices. Biochemistry 49:7665-7673.

91. Sengupta D, Prasanna X, Mohole M, Chattopadhyay A. 2018. Exploring GPCRlipid interactions by molecular dynamics simulations: excitements, challenges and the way forward. J. Phys. Chem. B. DOI: 10.1021/acs.jpcb.8b01657.

92. Sensi C, Daniele S, Parravicini C, Zappelli E, Russo V, Trincavelli ML, Martini C, Abbracchio MP, Eberini I. 2014. Oxysterols act as promiscuous ligands of class-A GPCRs: in silico molecular modeling and in vitro validation. Cell. Signal. 26:26142620.

93. Siani P, de Souza RM, Dias LG, Itri R, Khandelia H. 2016. An overview of molecular dynamics simulations of oxidized lipid systems, with a comparison of ELBA and MARTINI force fields for coarse grained lipid simulations. Biochim. Biophys. Acta 1858:2498-2511.

94. Swamy M, Beck-Garcia K, Beck-Garcia E, Hartl FA, Morath A, Yousefi OS, Dopfer EP, Molnár E, Schulze AK, Blanco R, Borroto A, Martín-Blanco N, Alarcon B, Höfer T, Minguet S, Schamel WW. 2016. A cholesterol-based allostery model of T cell receptor phosphorylation. Immunity 44:1091-1101.

95. Troupiotis-Tsaïlaki A, Zachmann J, González-Gil I, Gonzalez A, Ortega-Gutiérrez S, Lopez-Rodriguez ML, Pardo L, Govaerts C. 2017. Ligand chain length drives activation of lipid G protein-coupled receptors. Sci. Rep. 7:2020.

96. van Jaarsveld MT, Houthuijzen JM, Voest EE. Molecular mechanisms of target recognition by lipid GPCRs: relevance for cancer. 2016. Oncogene 35:4021-4035.

97. van Meer G. 2011. Dynamic transbilayer lipid asymmetry. Cold Spring Harb. Perspect. Biol. 3:a004671.

98. van Meer G, Voelker DR, Feigenson GW. 2008. Membrane lipids: where they are and how they behave. Nat. Rev. Mol. Cell Biol. 9:112-124.

99. Vernier PT, Ziegler MJ, Sun Y, Chang WV, Gundersen MA, Tieleman DP. 2006. Nanopore formation and phosphatidylserine externalization in a phospholipid bilayer at high transmembrane potential. J. Am. Chem. Soc. 128:6288-6289.

100. Volinsky R, Cwiklik L, Jurkiewicz P, Hof M, Jungwirth P, Kinnunen PK. 2011. Oxidized phosphatidylcholines facilitate phospholipid flip-flop in liposomes. Biophys. J. 101:1376-1384.

101. Wong-Ekkabut J, Xu Z, Triampo W, Tang IM, Tieleman DP, Monticelli L. 2007. Effect of lipid peroxidation on the properties of lipid bilayers: a molecular dynamics study. Biophys. J. 93:4225-4236.

102. Yin $\mathrm{H}, \mathrm{Xu}$ L, Porter NA. 2011. Free radical lipid peroxidation: mechanisms and analysis. Chem. Rev. 111:5944-5972.

103. Zacharias DA, Violin JD, Newton AC, Tsien RY. 2002. Partitioning of lipidmodified monomeric GFPs into membrane microdomains of live cells. Science 296:913-916.

104. Zocher M, Zhang C, Rasmussen SG, Kobilka BK, Müller DJ. 2012. Cholesterol increases kinetic, energetic, and mechanical stability of the human $\beta 2$-adrenergic receptor. Proc. Natl. Acad. Sci. USA 109:E3463-3472. 


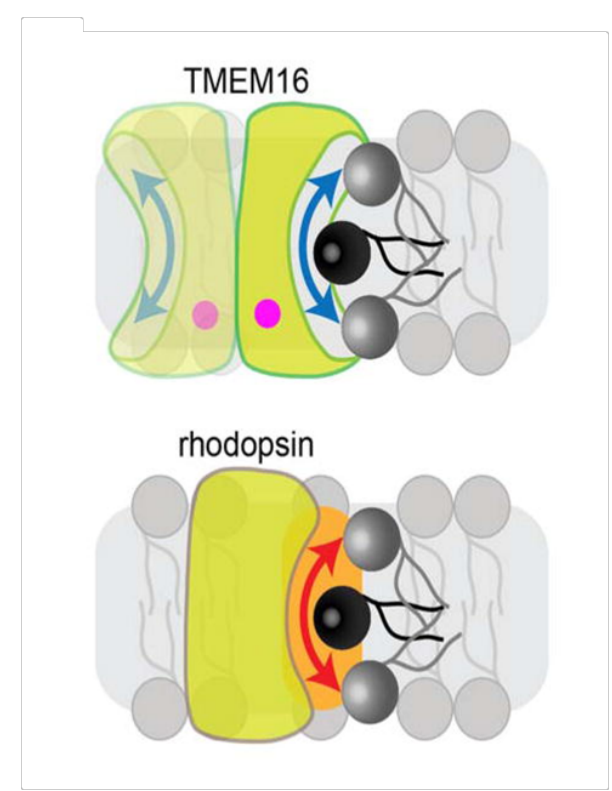

Figure 1. (Top) Homodimeric TMEM16 scramblase (containing bound $\mathrm{Ca}^{2+}$ indicated by pink dots) capable of scrambling a lipid along a hydrophilic groove facing the membrane, similar to swiping a credit card through a card reader. (Bottom) Rhodopsin-mediated lipid scrambling taking place along the rhodopsin-membrane interface (orange zone). Figure is adopted from ref. (21). 


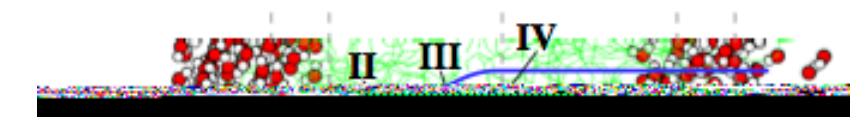

Figure 2. (Top) Translocation path next to the helices II, III, and IV of opsin. (Middle) Free energy profiles for POPC (blue) and cholesterol (red) translocation along the opsinmembrane interface. Statistical errors are depicted by the shaded area. The reaction coordinate $(z)$ is the difference between the lipid headgroup's center of mass and the bilayer center along the bilayer normal. (Bottom) Free energy profiles for POPC (blue) and cholesterol (red) translocation in a protein-free membrane. Figure is adopted from ref. (71). 


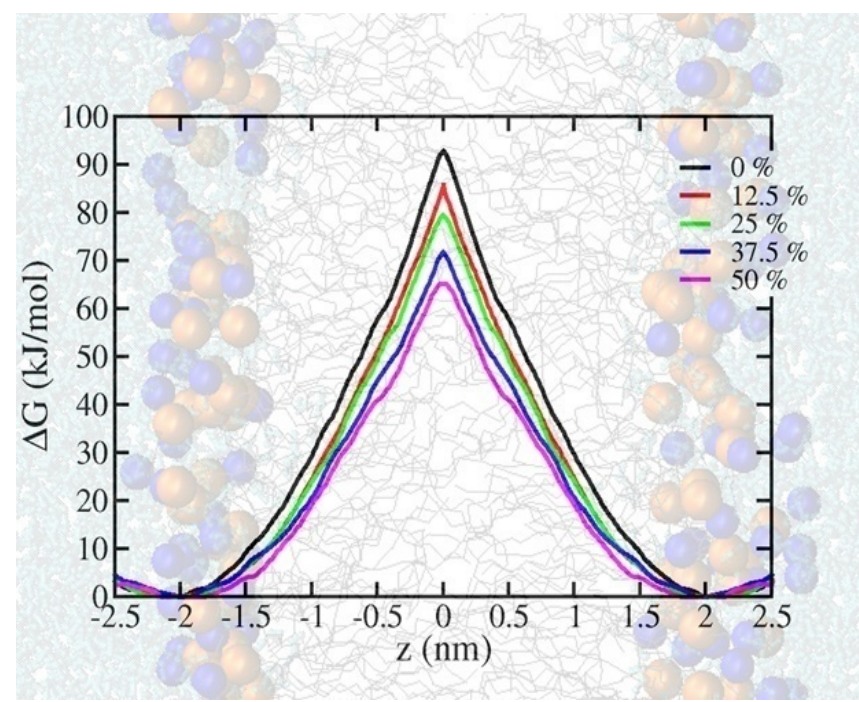

Figure 3. Free energy profiles for PS translocation through phospholipid bilayers with different levels of peroxidation (see inset). Figure is taken from ref. (83). 


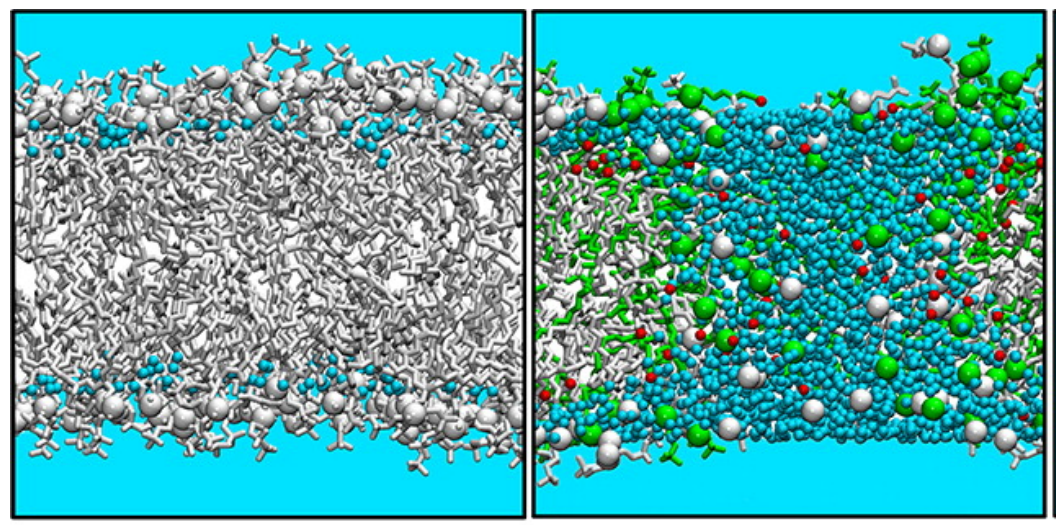

0

\section{Concentration of aldehyde lipids (\%)}

Figure 4. Pore formation and micellation in a lipid bilayer for an increasing concentration of aldehyde lipids (green) mixed with non-oxidized phospholipids (white). Green and white spheres represent the phosphorus atoms of the lipids. Red spheres depict the oxygen atom of the aldehyde group of the oxidized lipid tails. Blue spheres represent oxygen of water. Figure is taken from ref. (10). 


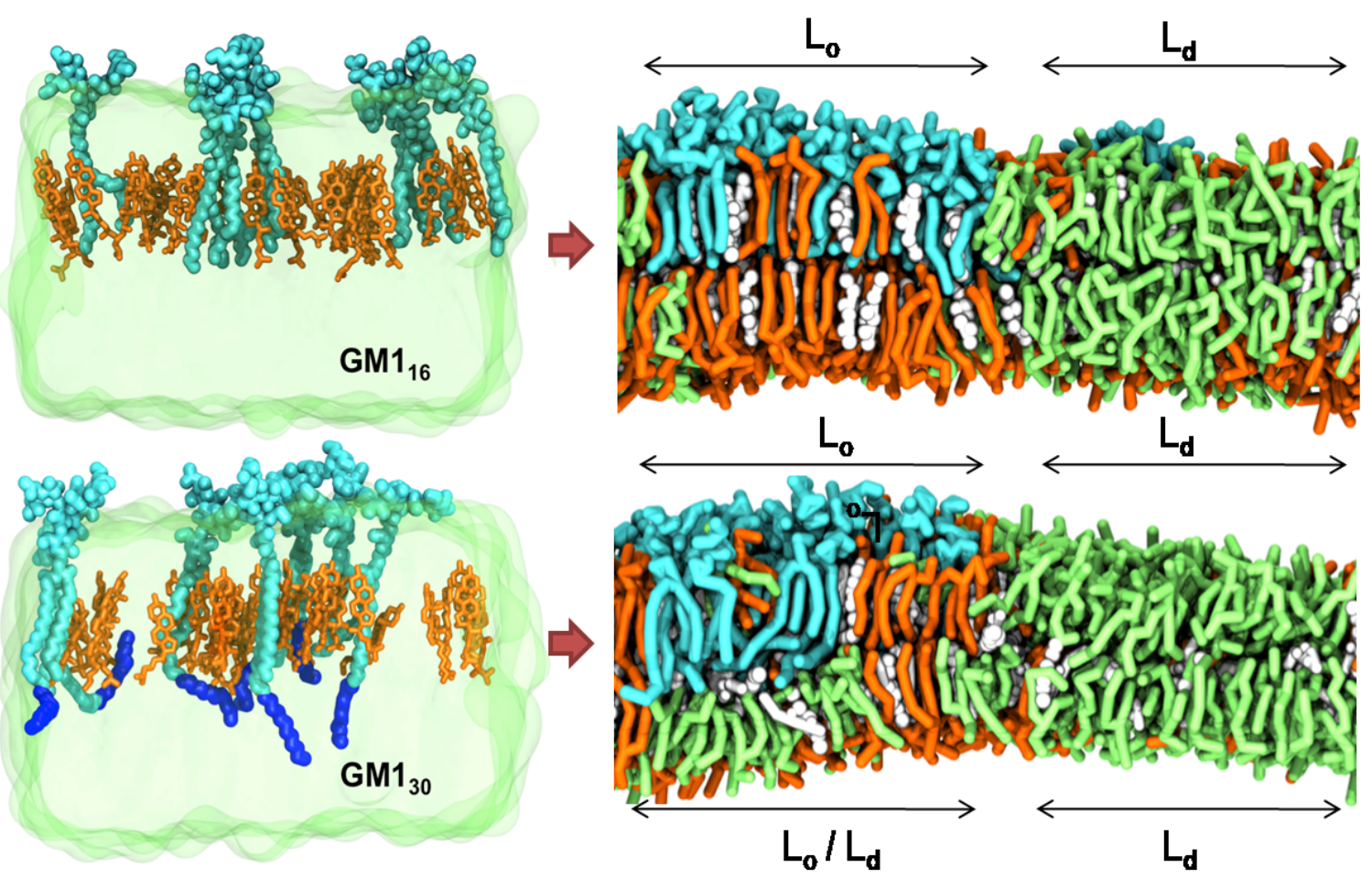

Figure 5. (Top) Transmembrane domain registration in a bilayer containing short-chain GM1. $\mathrm{L}_{\mathrm{o}}$ stands for the liquid-ordered phase (domain) and $\mathrm{L}_{\mathrm{d}}$ for the liquid-disordered phase. (Bottom) Mixing of saturated and unsaturated lipids in the inner leaflet induced by interdigitation of GM1 with long acyl chains. Figure is adopted from ref. (53). 


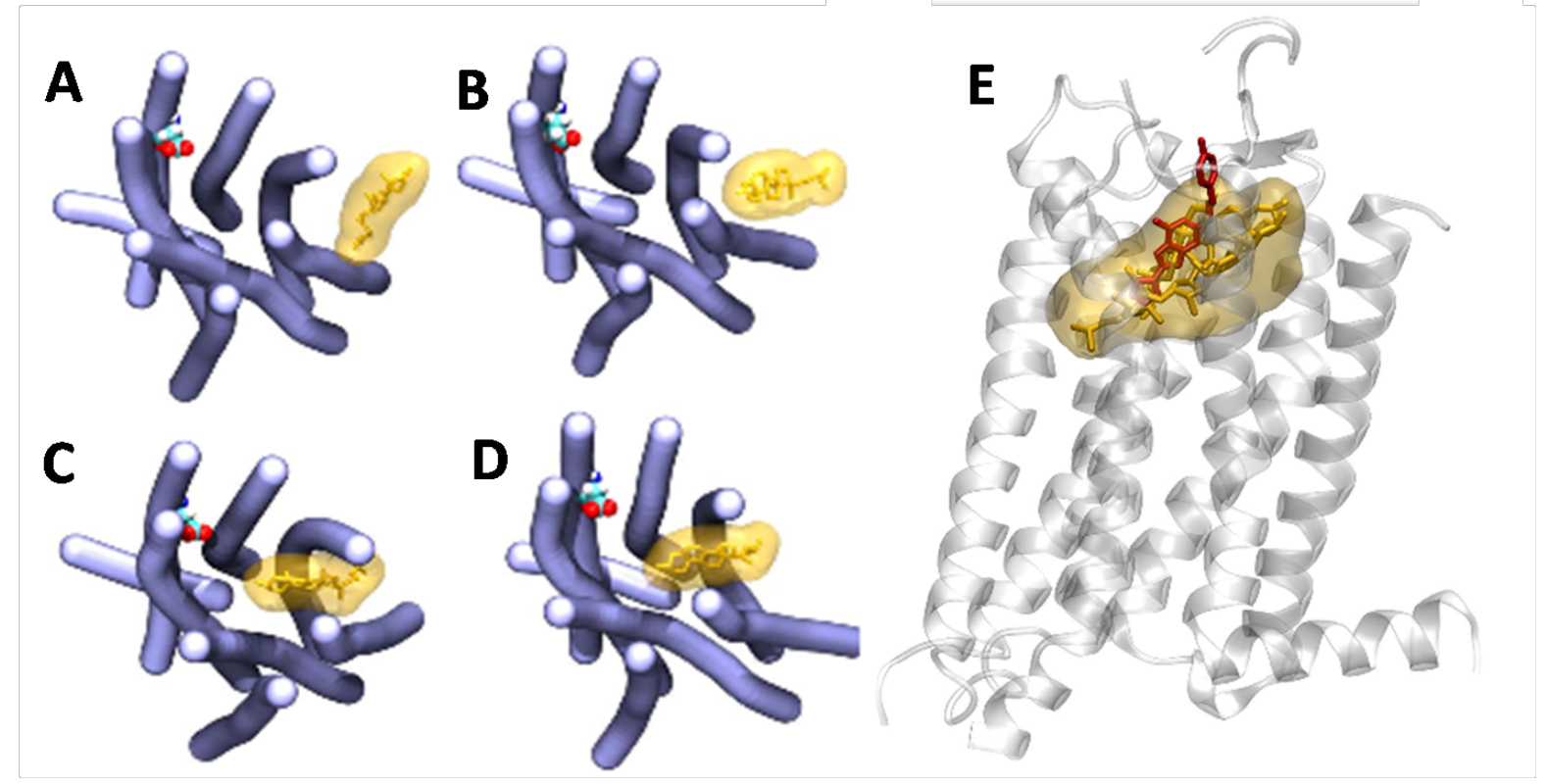

Figure 6. (A-D) Entrance of cholesterol (yellow) into the orthosteric ligand-binding site of $\mathrm{A}_{2 \mathrm{~A}} \mathrm{R}$ (blue). (E) The average position of cholesterol in the binding site calculated from simulations (a yellow transparent region) and superimposed on the crystal structure of the agonist-bound (red stick) receptor. Also shown is the final simulation snapshot of cholesterol at the binding site (yellow sticks). Figure is adopted from ref. (28). 